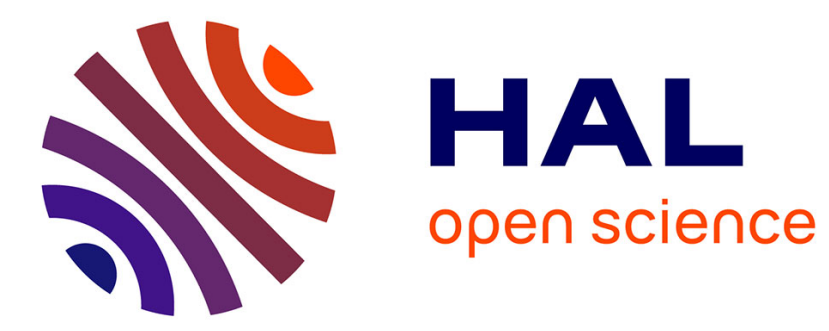

\title{
Microvascular blood flow monitoring with laser speckle contrast imaging using the generalized differences algorithm
}

Anne Humeau-Heurtier, Guillaume Mahé, Pierre Abraham

\section{- To cite this version:}

Anne Humeau-Heurtier, Guillaume Mahé, Pierre Abraham. Microvascular blood flow monitoring with laser speckle contrast imaging using the generalized differences algorithm. Microvascular Research, 2015, 98, pp.54 - 61. 10.1016/j.mvr.2014.12.003 . hal-01110640

HAL Id: hal-01110640

https://hal-univ-rennes1.archives-ouvertes.fr/hal-01110640

Submitted on 28 Jan 2015

HAL is a multi-disciplinary open access archive for the deposit and dissemination of scientific research documents, whether they are published or not. The documents may come from teaching and research institutions in France or abroad, or from public or private research centers.
L'archive ouverte pluridisciplinaire HAL, est destinée au dépôt et à la diffusion de documents scientifiques de niveau recherche, publiés ou non, émanant des établissements d'enseignement et de recherche français ou étrangers, des laboratoires publics ou privés. 


\title{
Microvascular blood flow monitoring
} with laser speckle contrast imaging using the generalized differences algorithm

\author{
Anne Humeau-Heurtier \\ University of Angers, LARIS - Laboratoire Angevin de Recherche en Ingénierie des \\ Systèmes, 62 avenue Notre-Dame du Lac, 49000 Angers, France \\ Guillaume Mahé \\ University of Rennes 1, CHU of Rennes, Pôle imagerie médicale et explorations \\ fonctionnelles, 35033 Rennes Cedex 9, France \\ Inserm, CIC 1414 "Ischemia, macro and microcirculation" group, 35033 Rennes Cedex 9, \\ France \\ Pierre Abraham \\ University of Angers, CHU of Angers, Laboratoire de Physiologie et d'Explorations \\ Vasculaires, UMR CNRS 6214-INSERM 1083, 49033 Angers cedex 01, France
}

\begin{abstract}
Laser speckle contrast imaging (LSCI) is a full-field optical technique to monitor microvascular blood flow with high spatial and temporal resolutions. It is used in many medical fields such as dermatology, vascular medicine, or neurosciences. However, LSCI leads to a large amount of data: image sampling frequency is often of several $\mathrm{Hz}$ and recordings usually last several minutes. Therefore, clinicians often perform regions of interest in which a spatial averaging of blood flow is performed and the result is followed with time. Unfortunately, this leads to a poor spatial resolution for the analyzed data. At the same time, a higher spatial resolution for the perfusion maps is wanted. To get over this dilemma we propose a new post-acquisition visual representation for LSCI perfusion data using the so-called generalized differences (GD) algorithm. From a stack of
\end{abstract}

\footnotetext{
* Corresponding author

Email address: anne.humeau@univ-angers.fr (Anne Humeau-Heurtier)
} 
perfusion images, the procedure leads to a new single image with the same spatial resolution as the original images and this new image reflects perfusion changes. The algorithm is herein applied on simulated stacks of images and on experimental LSCI perfusion data acquired in three different situations with a commercialized laser speckle contrast imager. The results show that the GD algorithm provides a new way of visualizing LSCI perfusion data.

Keywords: Medical and biological imaging, Laser speckle, Image analysis, Blood flow, Microcirculation

\section{Introduction}

The monitoring of microvascular blood flow can be performed with several optical techniques, among which laser Doppler flowmetry (LDF) and laser speckle contrast imaging (LSCI) are now currently used [1]. In LDF, the tis5 sue under study (skin for example) is illuminated with a low-power laser light. The backscattered light is transmitted to a photodetector. LDF relies on the Doppler frequency shift that appears when light is scattered by moving blood cells (mainly red blood cells). The LDF perfusion is defined from the first moment of the power spectrum of the photocurrent fluctuations (see, e.g., $[2,3]$ ). LDF signals have been the subject of many works (see, e.g., Refs. [4, 5]), but one of the major drawbacks of LDF is that it is a single-point measurement technique. Due to the spatial variations of the microvascular blood flow $[6,7]$, the reproducibility of the measure is poor $[8,9]$. Laser Doppler imaging (LDI) has been designed to prevent this drawback [10]. However, for most of imagers, the image is computed by scanning the area under study which entails long recording times and prevents the monitoring of rapid physiological phenomena. To overcome the latter point, full-field laser Doppler imagers have recently been proposed $[11,12,13,14,15,16,17,18]$, but require high-speed cameras.

LSCI is another full-field optical technique for the monitoring of microvascular blood flow. It is a real-time method that does not need any scan and uses a normal CCD or CMOS camera [19]. LSCI relies on the following prin- 

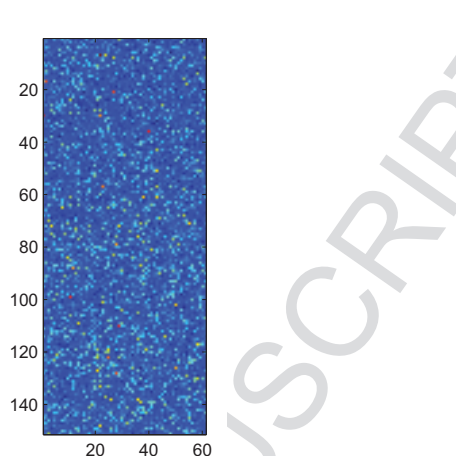

Figure 1: Perfusion image (151 rows and 61 columns) from LSCI technique of a zone on the forearm of a healthy subject.

ciple $[20,21,22,23,24,25,26,27,28,29]$ : when the tissue under study is illuminated by a laser with an expanded beam, the backscattered light forms an interference pattern on the detector (a camera). Due to phase difference involved in the backscattered light, there are constructive and destructive interferences. The latter produce a pattern composed of bright and dark areas on the camera. This pattern is known as a speckle pattern. When particles move in the tissue under study, the speckle pattern changes. In LSCI, the changing speckle pattern is recorded with a camera that has an integration time in the millisecond range. Due to the long integration time compared to the typical decorrelation time of the speckle pattern, the speckle pattern is blurred in the image. The level of blurring is quantified by the speckle contrast $K$ that is inversely related to blood flow (see an example in Fig. 1). In the laser speckle contrast imager used in our work, the perfusion is computed from $1 / K-1$ [30]. It has been shown that the power spectral density measurements of the light fluctuations derived using LSCI and LDF techniques are equivalent [31].

LSCI data have shown to have excellent reproducibility $[9,32,33,34]$. The rapid adoption of LSCI in clinical research is also probably due to the relative 40 ease and low cost of building an instrument, compared to other techniques such 
as MRI or CT [19, 35]. The developments of the technique are again the subject of many studies (see, e.g., [36],[37],[38, 39, 40],[41],[42, 43, 44, 45, 46, 47],[48]). LSCI is now used in many medical fields such as dermatology, cardiology, vascular medicine, diabetology, neuroscience, ophthalmology [26], among others. In cardiovascular studies, LSCI can be used to analyze the impairment of tissue blood supply provoked by pathologies such as diabetes, Raynaud's phenomenon, or peripheral vascular diseases. Monitoring blood flow with LSCI can therefore allow early diagnoses or an evaluation of the evolution for such diseases. However, LSCI (as LDF and LDI) gives blood flow values in arbitrary units: no absolute values as $\mathrm{ml} \cdot \mathrm{g}^{-1} \cdot \mathrm{min}^{-1}$ tissue are possible [22, 49], as again pointed out recently (see, e.g., [29]). Another drawback of the LSCI technique is that it leads to a large amount of data: the frequency sampling of the images can be of several $\mathrm{Hz}$ (depending on the application) and the recordings usually last several minutes. In order to monitor the blood flow, the clinicians have therefore a stack containing many images (perfusion maps) reflecting the temporal evolution of the blood flow on the area under study. To analyze the data easily and rapidly, one or several regions of interest (ROIs) are often chosen on the first image of the image stack, and an average of the blood flow is performed in this ROI. The same ROI is chosen on the other images of the stack and thus an average of the blood flow can be studied in this ROI in time, at the rate of the image frequency acquisition $[26,32]$. This leads to a 1D-signal reflecting the time evolution of the average value for the blood flow in the ROI (see an example in Fig. 2). In the same time, algorithms or methods to obtain a higher spatial resolution for the images are still proposed (see, e.g., [50, 51, 52, 53]). However, this spatial resolution is "lost" by the averaging procedure performed in the ROIs, in clinical routine, to evaluate the evolution of the blood flow in a simple and rapid way. To get over this dilemma we propose herein a new way of visualizing LSCI perfusion data from commercialized imagers. Our procedure uses the so-called generalized differences (GD) algorithm. This leads to the computation of a new single perfusion image reflecting by itself the variations of the blood flow on the whole images of the stack. Moreover, this new im- 


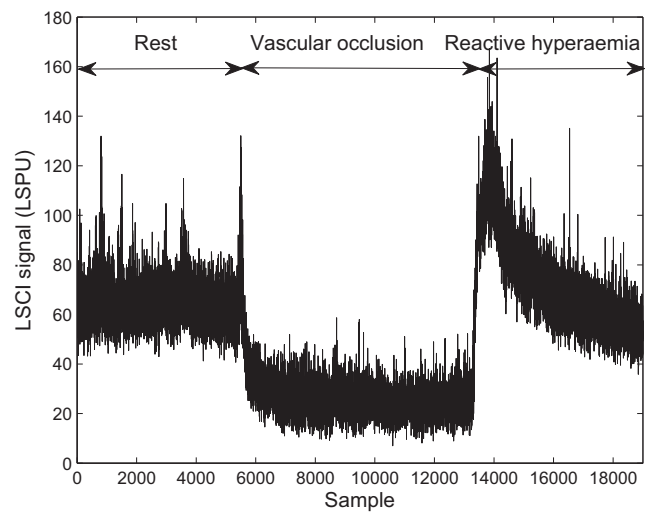

Figure 2: Laser speckle contrast signal (perfusion signal) of a zone on the forearm of a healthy subject, at rest, during vascular occlusion and post-occlusive reactive hyperaemia, computed from spatial averaging on a region of interest of $7 \times 7$ pixels $^{2}$ in laser speckle contrast images (perfusion images).

age has the advantage of possessing the same spatial resolution as the original images. Our work therefore corresponds to a post-acquisition perfusion image processing procedure. Our goal is not to propose an imaging pre-processing method on raw speckle images. The problem raised by large volumes of data when imaging blood flow is of importance. Moreover, the visualization of blood flow data remains a challenging task (see, e.g., [54, 55]).

Among the algorithms that are often used in the evaluation of the activity for laser speckle images we find the Fujii's method $[56,57]$ and the temporal difference method [58]. However, unlike the GD method (see below), the Fujii's algorithm does not take into account the difference between nonconsecutive images. This presents a drawback in the case of laser speckle perfusion evaluation as variations of perfusion are slow compared to the frequency of the image acquisition; the variations are therefore low between two consecutive images. Comparisons between perfusion images differing by short times are not reliable because this prevents a salient analysis of slow phenomena when a relatively 
short duration is studied (a few seconds). Moreover, as for the Fujii's method, the temporal difference method is not appropriated for microvascular blood flow characterization with laser speckle perfusion data because the difference between nonconsecutive perfusion images are not taken into account. Moreover, the temporal difference method leads to a stack of $N-1$ difference images which does not correspond to our goal to obtain a new single image. These two methods have therefore been discarded from our work.

In what follows, we first present the GD algorithm. Afterwards, the proof of concept of our work and the measurement procedure used to acquire experimental laser speckle contrast images in different situations are described. The results obtained on simulated and experimental data with the GD algorithm are detailed and discussed. Moreover, the results are compared with those given by other algorithms.

\section{Materials and Methods}

\subsection{Generalized differences}

In the method called generalized differences, a new image $P U_{G D}$ is constructed from a sequence of $N$ (perfusion) images $P U_{k}(1 \leq k \leq N)$ as [59]

$$
P U_{G D}(i, j)=\sum_{k=1}^{N-1} \sum_{l=k+1}^{N}\left|P U_{k}(i, j)-P U_{l}(i, j)\right|,
$$

where $P U_{k}(i, j)$ is the perfusion value for the pixel situated at the coordinates $(i, j)$ in the $k$-th image of the perfusion sequence (a similar notation is used for $\left.P U_{l}(i, j)\right)$. In the new image $P U_{G D}$, regions differing in their activity are displayed as different gray levels. From Eq. 1, we observe that the GD algorithm does not depend on the order of the images. Moreover, the pixel $(i, j)$ in image $P U_{G D}$ is zero (minimum value) when all the $P U_{k}(i, j)$ values are equal (no movement at this pixel). 
Another variant (denoted as GD*) considers not absolute values but square of $20,40,60,45,25$ on the square of images $5,6,7,8$ and 9 respectively. We then applied the GD algorithm on the stack of 15 images with the aim to see if it is able to detect the transient variation of pixel values. 


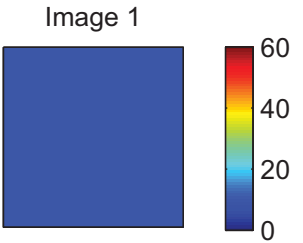

Image 6

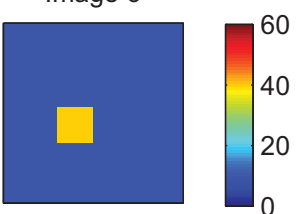

Image 8

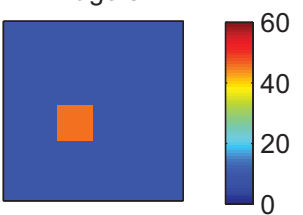

Image 5

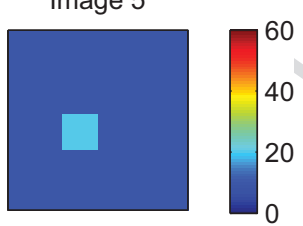

Image 7

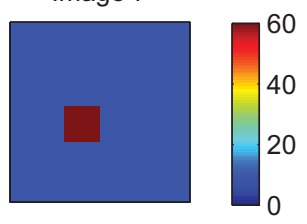

Image 9

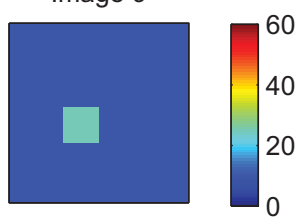

Figure 3: Representation of images 1, 5, 6, 7, 8, and 9 of the simulated images (see text for details).

We then generated another stack of images in order to analyze the influence of possible experimental noise on the resulting GD image. For LSCI, the experimental noise usually arise from movement. For this purpose, we generated 15 simulated images, $30 \times 30$ pixels $^{2}$ each. On these 15 images, all the pixel values are identical and equal to 10 (simulating blood flow with no variations at all, or no blood flow (biological zero)), except on images 5 to 9 where a square of size $6 \times 6$ pixels $^{2}$ had the same values as previously (see Fig. 3), simulating a transient increase of blood flow in this region of interest. Moreover, on images 4 to 8 , three smaller squares $\left(4 \times 4\right.$ pixels $\left.^{2}\right)$ of different amplitudes have been inserted: the first square contains pixels with random amplitudes varying from 25 to 27 . The second square contains pixels with random amplitudes varying from 45 to 55, whereas the third square contains pixels with random amplitudes varying from 200 to 220. Different random values were generated for each of the images 4 to 8 . These three "noisy" squares have therefore pixel amplitudes 

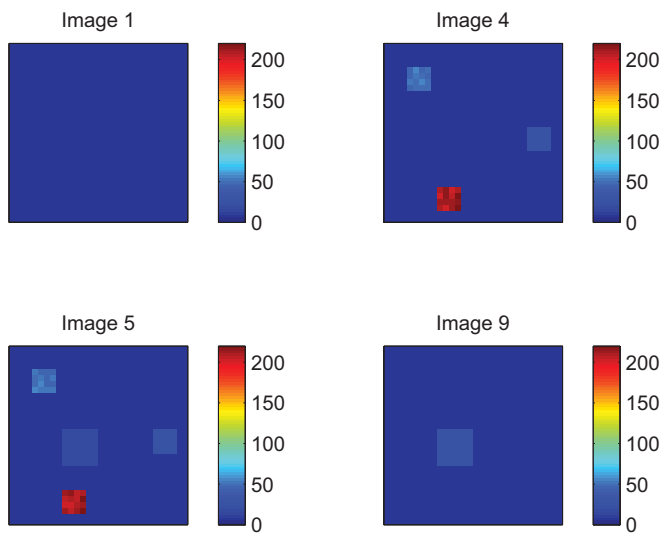

Figure 4: Representation of images 1, 4, 5, and 9 of the simulated images used to analyze the influence of movement artefacts (see text for details).

\section{increase of blood flow, simulating transient increases of pixel amplitude due to}

varying from half to more than four times larger than the simulated transient movement artefacts (see Fig. 4).

\subsection{Experimental image acquisition}

Three kinds of experimental LSCI recordings have been performed to evaluate the performance of the GD algorithm in the visualization of perfusion changes from real LSCI data. All the image acquisitions have been done in laser speckle perfusion units ( $L S P U)$ with a PeriCam PSI System (Perimed, Sweden) having a laser wavelength of $785 \mathrm{~nm}$ and an exposure time of $6 \mathrm{~ms}$.

The first recording has been performed on the two hemispheres of a mouse, recorded through the skull. The experiment has been performed in compliance with institutional guidelines and international standards on animal welfare and approved according to local and national regulation for animal care and use for research purposes [63]. The mouse was anesthetized with isoflurane $(2.5 \%$ 
induction, 1.5-2\% during surgery) in $70 \% \mathrm{~N} 2 \mathrm{O}$ and $30 \% \mathrm{O} 2$. After end of preparation isoflurane anesthesia was reduced to $1.1-1.2 \%$. Temperature was continuously measured and maintained at $37.8^{\circ} \mathrm{C}$ by use of an automatic controlled homeothermic blanket system. The mouse was placed in a stereotaxic frame and the skin was removed above both hemispheres to enable cerebral blood flow measurements by LSCI. At the parietal bone a small craniotomy ( $\sim 3 \mathrm{~mm}$ lateral from midline/lambda and rostral to the lambdoid suture; diameter: $\sim 1.2 \mathrm{~mm}$ ) was made by use of a dental drill (SI-923 implantMED, W\&H Deutschland GmbH, Laufen Germany) for spreading depression induction. The LSCI recordings have been performed with a sampling frequency of $0.1 \mathrm{~Hz}$ and the distance between the laser head to skull was set at $10.4 \mathrm{~cm}$ [64] which gave images with a resolution around $0.02 \mathrm{~mm}$. The provocation used to obtain perfusion variations was a pinprick in one of the hemispheres that induced a cortical spreading depression/depolarization (CSD). CSD is a self-propagating wave of depolarization which corresponds to a slow-moving ionic and metabolic disturbance that propagates in cortical brain tissue [65]. The perfusion map stack processed was composed of 10 images.

The second experimental LSCI recordings have been performed on the forearm skin of a healthy subject. The variations of the perfusion have been induced by a local heating. The latter leads to a vasodilation and therefore to an increase of the perfusion values $[66,67,68]$. The recordings have been performed with a sampling frequency of $5 \mathrm{~Hz}$ and the distance between the laser head to skin was set at $14.7 \mathrm{~cm}$ [64] which gave images with a resolution around $0.14 \mathrm{~mm}$. The perfusion map stack processed was composed of 100 images.

195

The third experimental LSCI recordings have been performed on the forearm skin of another healthy subject. The variations of the perfusion have been induced by a local pinching. The latter was manually done and performed on the ventral face of the forearm. The pinching induced a local and transient 


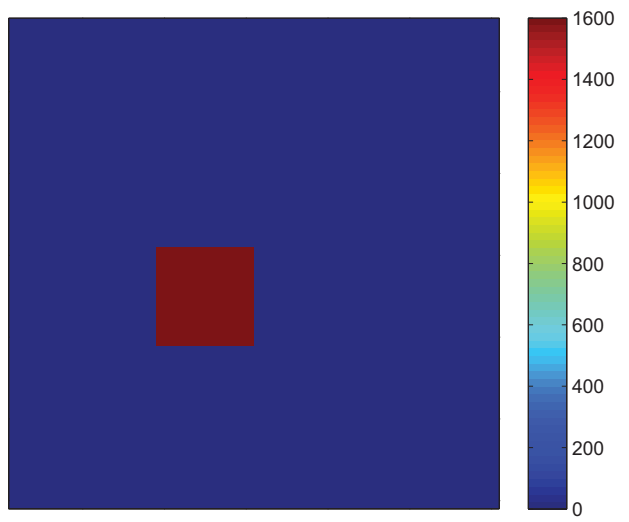

Figure 5: Image computed with the generalized differences algorithm from 15 simulated images (see Fig. 3).

frequency of $5 \mathrm{~Hz}$ and the distance between the laser head to skin was set at $15.9 \mathrm{~cm}$ [64] which gave images with a resolution around $0.15 \mathrm{~mm}$. The perfusion map stack processed was composed of 100 images.

\section{Results and Discussion}

\subsection{Simulated images}

The result of the GD algorithm for the simulated data shown in Fig. 3 is represented in Fig. 5. From the latter, we observe that the GD image is zero everywhere except in the square that was present in images 5 to 9 of the stack. In the new image $P U_{G D}$, the pixels are zero (minimum value) when all the pixel values are equal in the stack (no movement at this pixel) and regions differing in their activity are displayed as different gray levels. This result is therefore a proof of our concept for the use of the GD algorithm to detect perfusion changes in LSCI data. 


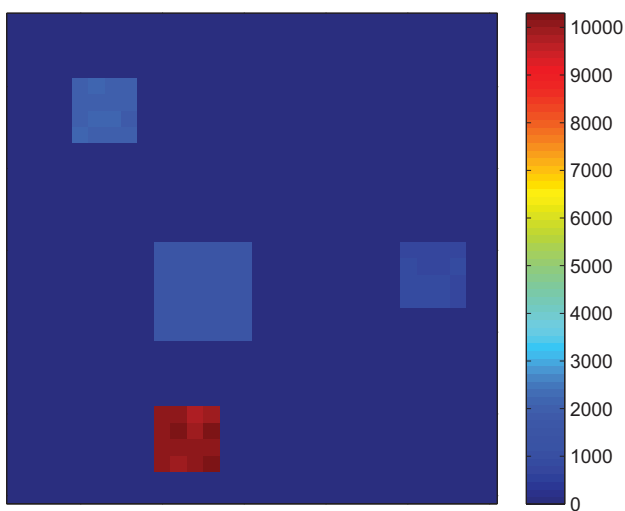

Figure 6: Image computed with the generalized differences algorithm from 15 simulated images (see Fig. 4).

The results of the GD algorithm for the stack of 15 images containing movement artefacts (Fig. 4) is shown in Fig. 6. The results show that the image generated with the GD algorithm reflects all the variations: all the four squares are visible in the new image, the one due to the transient increase of blood flow and the three squares reflecting the movement artefacts. Therefore, the GD algorithm keeps the transient increase of blood flow visible, but also reflects pixel amplitude variations due to movement artefacts. However, LSCI is by definition very sensitive to movements. That is why LSCI experiments have to be performed with still subjects to avoid experimental noise.

\subsection{Experimental images}

The first perfusion image of the recordings for the three experimental cases is presented in Figs. 7 to 9. The results of the GD algorithm for the three cases are shown in Figs. 10 to 12. For each of these latter figures, we can observe in a single frame where the perfusion has changed (due to the stimulus). For 


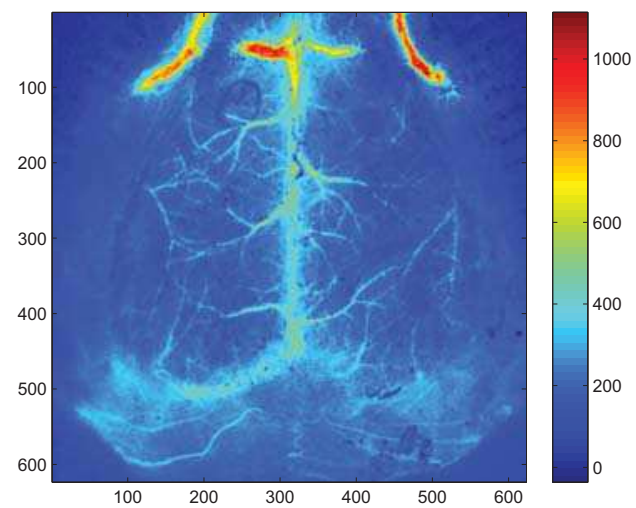

Figure 7: Perfusion map (623 rows and 623 columns) representing the two hemispheres of a mouse, recorded through the skull [63].

part of the image. For case 2 (see Fig. 11), we observe that the variations of the perfusion due to the local heating mostly appear in the bottom left part of the image. Finally, for case 3 (see Fig. 12), the perfusion variations are globally localized on all the image. As mentioned above, Fig. 10 has been computed from 10 images and Figs. 11 and 12 have been computed from 100 images.

Due to the spatial heterogeneity of the microcirculation $[6,7]$ and because clinical cases require perfusion analyses with a high spatial resolution (see, e.g., Ref. [69]), LSCI is an interesting optical technology. However, the visualization of LSCI perfusion images should be easy and rapid. Due to the high amount of images generated by several minutes of recordings, the GD image is an interesting way to visualize the variations of the perfusion (post-acquisition procedure) with a spatial resolution as high as the original perfusion maps.

As shown in Fig. 6, the GD image reflects perfusion changes but also movement artefacts. This is also shown in Fig. 13 where the GD image has been computed from perfusion data recorded on the forearm of a healthy subject during a local heating while movements were performed by the subject. We 


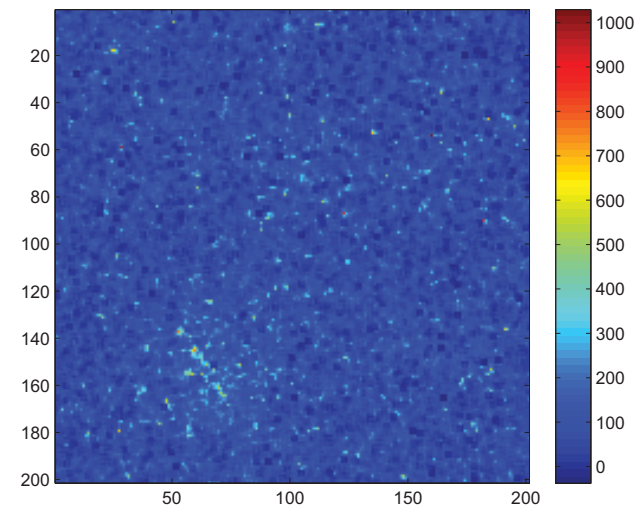

Figure 8: Perfusion map (201 rows and 201 columns) representing the forearm skin at the very beginning of a local heating.

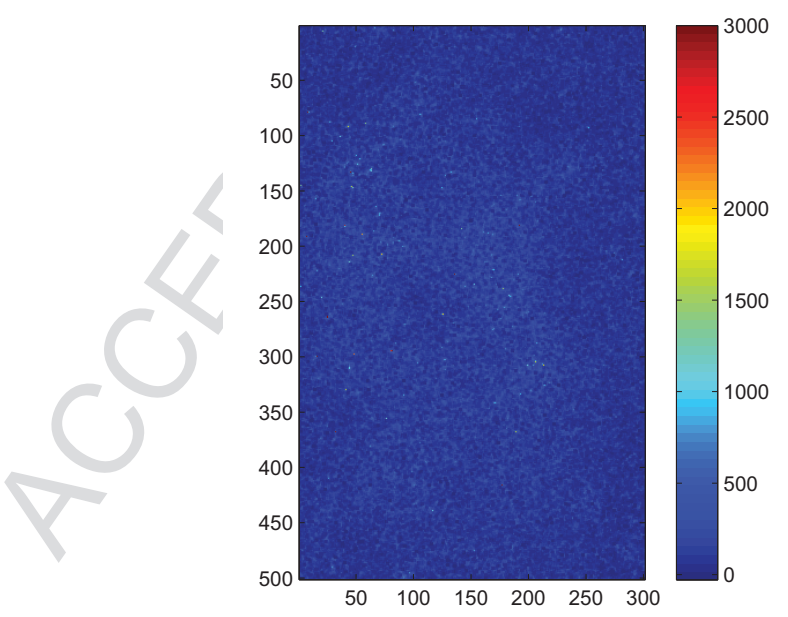

Figure 9: Perfusion map (501 rows and 301 columns) representing the forearm skin just after a local pinching.

note that both perfusion changes and movement artefacts lead to high pixel

values in the GD image. In order to avoid misinterpretation between perfusion changes and movement artefacts, the acquisitions have to be performed when 


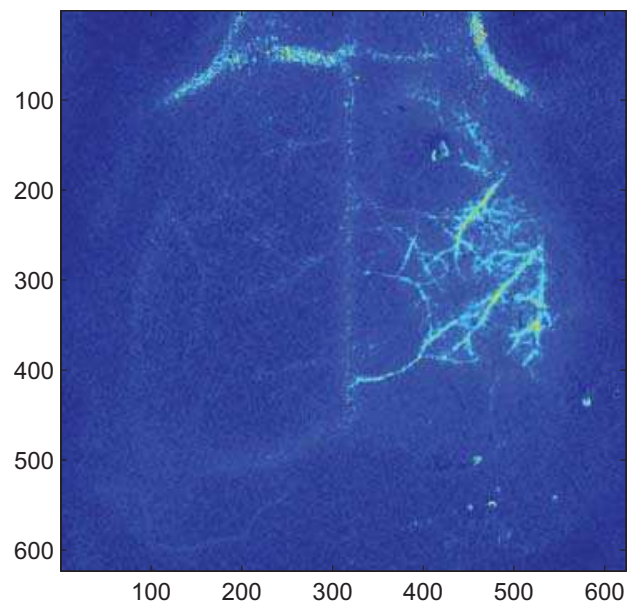

Figure 10: Image (623 rows and 623 columns) computed with the generalized differences algorithm from 10 perfusion images recorded during a pinprick in one of the hemispheres that induces a wave of ischemia (see text for details).

the subject is totally still.

We also compared our results given by the GD algorithm on the experimental LSCI data with the ones given by the GD^ algorithm and by the Fujii's method. The results given by the GD* algorithm are shown in Figs. 14 to 16 . The ones given by the Fujii's method are shown in Figs. 17 to 19. From these figures, we observe that the perfusion changes (due to the stimulus) are much less visible than the ones given by the GD algorithm. The GD algorithm therefore appears as the most interesting. No comparison with the temporal difference method is possible because, with the latter method, a sequence of $N$ images leads to a new sequence of $N-1$ difference images, and this does not corresponds to our goal.

For other dynamic vascular phenomena, it could be possible to compute the new GD image on more, or less, images in order to visualize slow or rapid phe- 


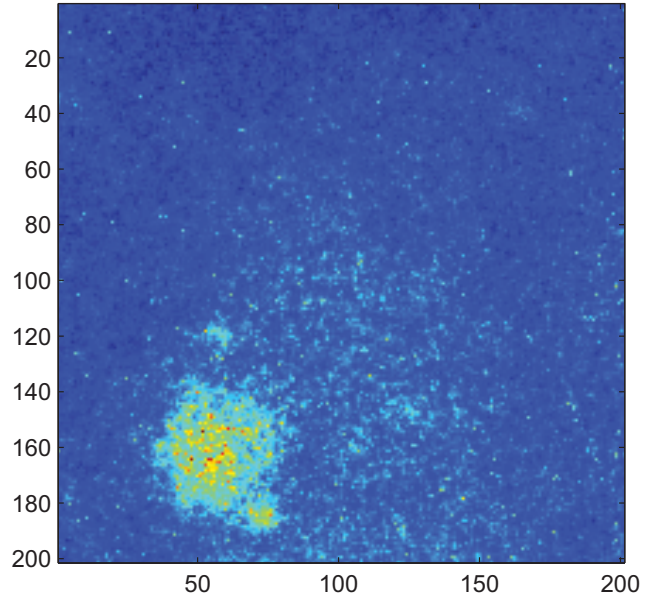

Figure 11: Image (201 rows and 201 columns) computed with the generalized differences algorithm from 100 perfusion images recorded during and after a local heating (see text for details).

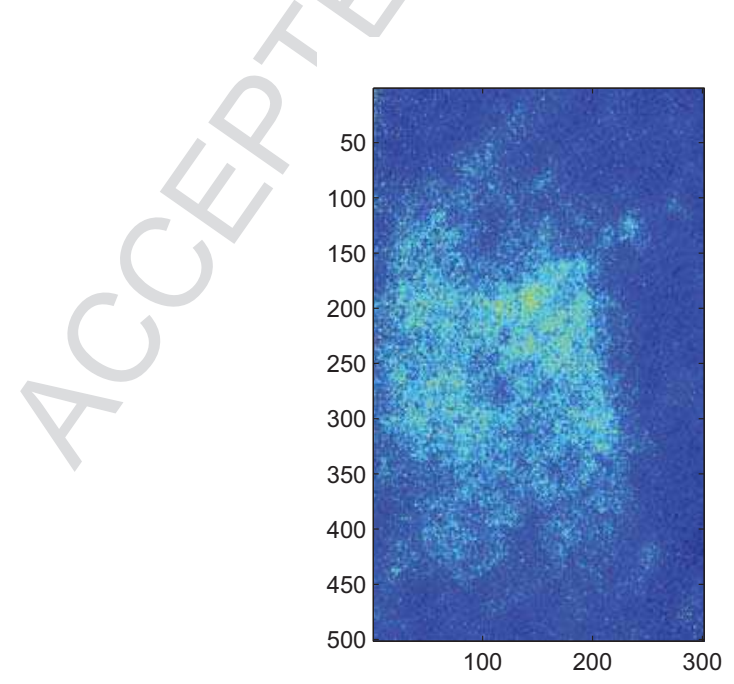

Figure 12: Image (501 rows and 301 columns) computed with the generalized differences algorithm from 100 perfusion images recorded just after a local pinching (see text for details). 


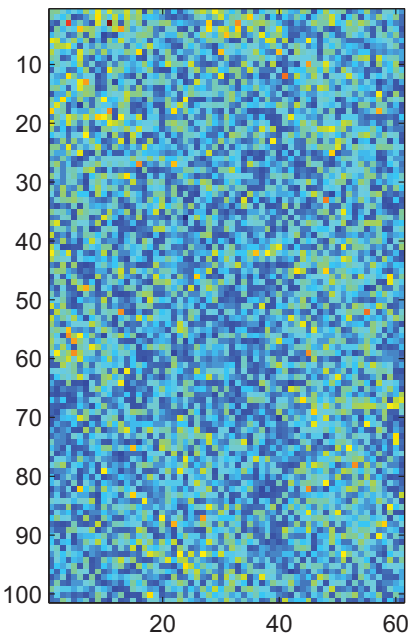

Figure 13: Image (101 rows and 61 columns) computed with the generalized differences algorithm from 100 perfusion images recorded during and after a local heating, while movements were performed by the subject (see text for details).

nomena. A normalization could be useful to compare results coming from GD calculated from different numbers of images.

Moreover, by definition, the GD algorithm loses the time dimension. Therefore, it is not directly possible to obtain a GD measure in time. If one wants to obtain information on the time evolution of the perfusion changes, it could be possible to split the stack of images under study in several sub-stacks of images and to compute the GD image for each sub-stack. However, this splitting would lead to several GD images. Therefore, the number of sub-stacks should not be too large as this would lead to many GD images and would prevent an easy and rapid examination of perfusion changes.

The visualization of perfusion data remains a challenging task and our work 280 is the first one that proposes a post-acquisition procedure for visualizing LSCI 


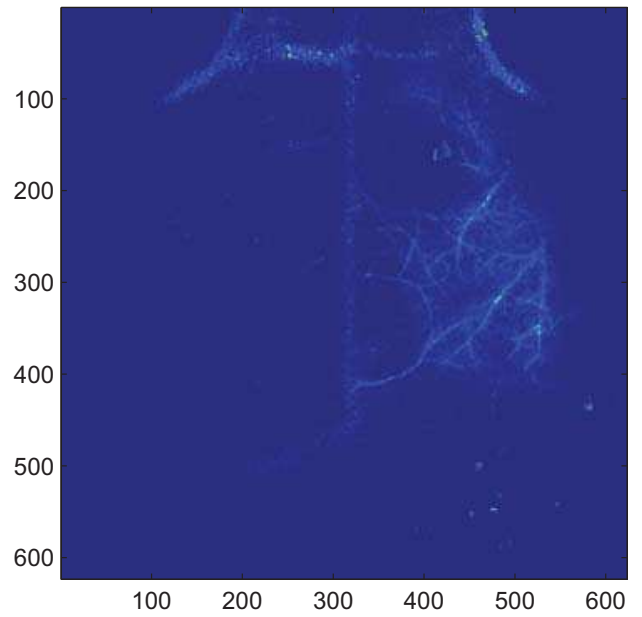

Figure 14: Image (623 rows and 623 columns) computed with the modified generalized differences algorithm $\left(\mathrm{GD}^{\star}\right)$ from 10 perfusion images recorded during a pinprick in one of the hemispheres that induces a wave of ischemia (see text for details).

perfusion maps coming from commercialized imagers. The GD algorithm appears as being an interesting tool in the evaluation of blood flow with high spatial resolution, which is of the utmost interest for clinical cases.

\section{Acknowledgment}

The authors would like to thank N. Offenhauser and J. Dreier for the recordings performed on the mouse.

\section{References}

[1] A. Humeau-Heurtier, E. Guerreschi, P. Abraham, and G. Mahé, "Relevance of laser Doppler and laser speckle techniques for assessing vascular function: state of the art and future trends," IEEE Trans. Biomed. Eng. 60, 659-666 (2013). 


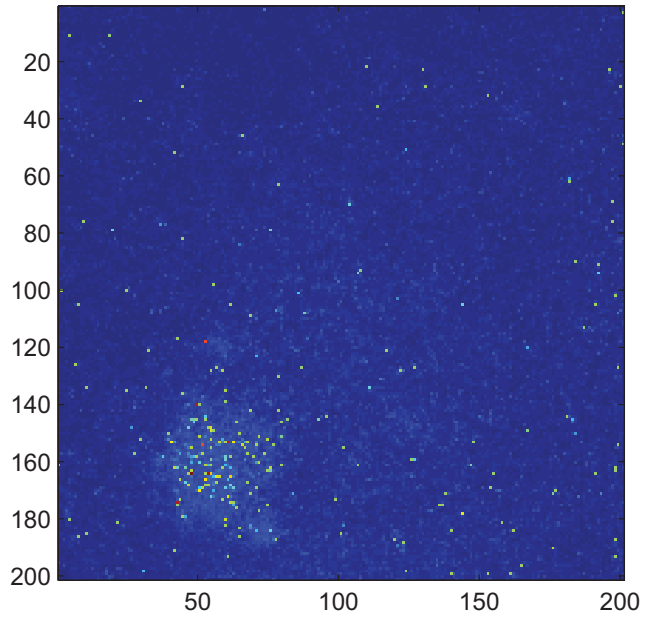

Figure 15: Image (201 rows and 201 columns) computed with the modified generalized differences algorithm $\left(\mathrm{GD}^{\star}\right)$ from 100 perfusion images recorded during and after a local heating (see text for details).

[2] M. D. Stern, "In vivo evaluation of microcirculation by coherent light scattering," Nature 254, 56-58 (1975).

[3] A. Humeau, W. Steenbergen, H. Nilsson, and T. Stromberg, "Laser Doppler perfusion monitoring and imaging: novel approaches," Med. Biol. Eng. Comput. 45, 421-435 (2007).

[4] A. Stefanovska, M. Bracic, and H.D. Kvernmo, "Wavelet analysis of oscillations in the peripheral blood circulation measured by laser Doppler technique," IEEE Trans. Biomed. Eng. 46, 1230-1239 (1999).

[5] A. Bernjak, G.A. Deitrick, W.A. Bauman, A. Stefanovska, and J. Tuckman, "Basal sympathetic activity to the microcirculation in tetraplegic man revealed by wavelet transform of laser Doppler flowmetry," Microvasc Res. 81, 313-318 (2011).

[6] K. Wardell, I. M. Braverman, D. G. Silverman, and G. E. Nilsson, "Spatial 


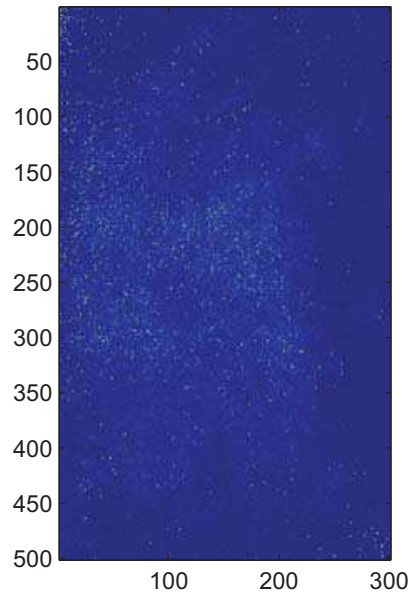

Figure 16: Image (501 rows and 301 columns) computed with the modified generalized differences algorithm $\left(\mathrm{GD}^{\star}\right)$ from 100 perfusion images recorded just after a local pinching (see text for details). heterogeneity in normal skin perfusion recorded with laser Doppler imaging and flowmetry," Microvasc. Res. 48, 26-38 (1994).

[7] I. M. Braverman, "The cutaneous microcirculation," J. Investig. Dermatol. Symp. Proc. 5, 3-9 (2000).

[8] M. Roustit, S. Blaise, C. Millet, and J. L. Cracowski, "Reproducibility and methodological issues of skin post-occlusive and thermal hyperemia assessed by single-point laser Doppler flowmetry," Microvasc. Res. 79, 102$108(2010)$.

[9] M. Roustit, C. Millet, S. Blaise, B. Dufournet, and J. L. Cracowski, "Excellent reproducibility of laser speckle contrast imaging to assess skin microvascular reactivity," Microvasc. Res. 80, 505-511 (2010).

[10] K. Wardell, A. Jakobsson, and G. E. Nilsson, "Laser Doppler perfusion imaging by dynamic light scattering," IEEE Trans. Biomed. Eng. 40, 309316 (1993). 


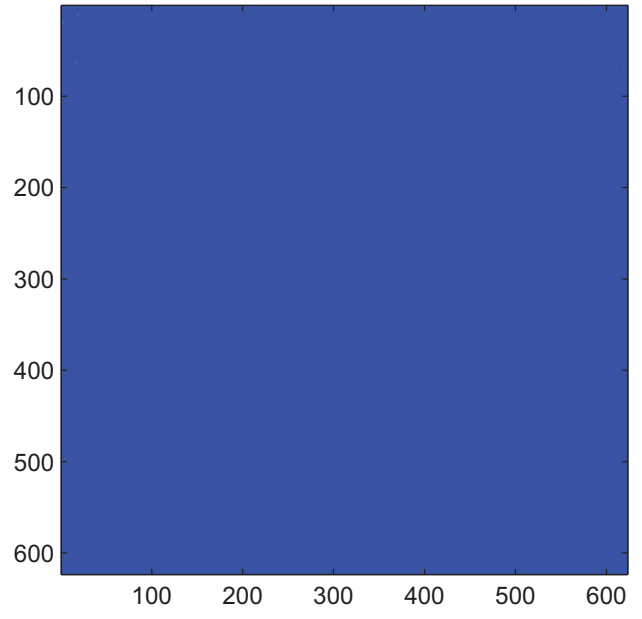

Figure 17: Image (623 rows and 623 columns) computed with the Fujii's method from 10 perfusion images recorded during a pinprick in one of the hemispheres that induces a wave of ischemia (see text for details).

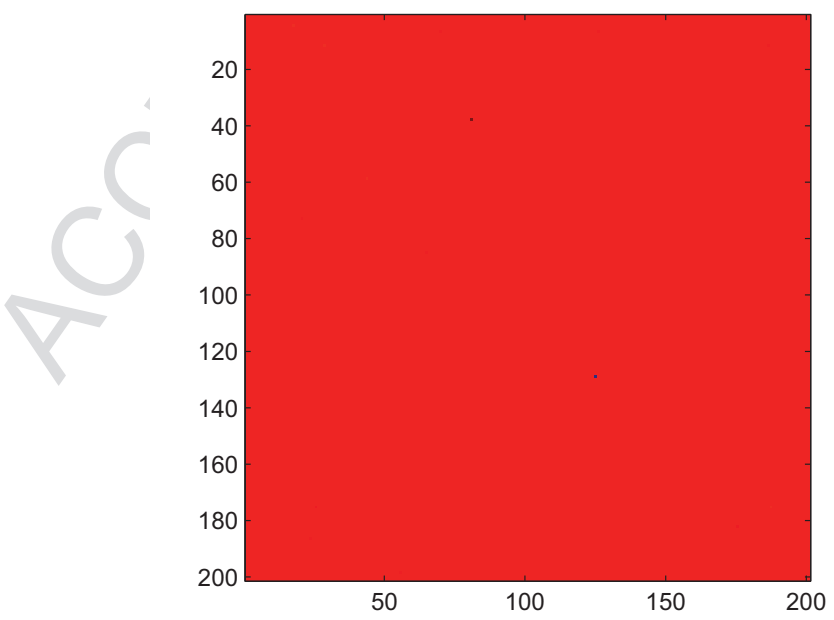

Figure 18: Image (201 rows and 201 columns) computed with the Fujii's method from 100 perfusion images recorded during and after a local heating (see text for details). 

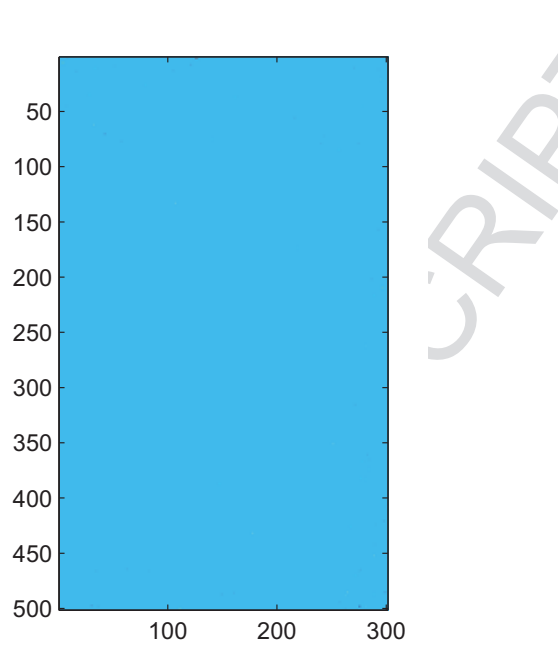

Figure 19: Image (501 rows and 301 columns) computed with the Fujii's method from 100 perfusion images recorded just after a local pinching (see text for details).

[11] A. Serov, W. Steenbergen, and F. de Mul, "Laser Doppler perfusion imaging with a complimentary metal oxide semiconductor image sensor," Opt. Lett. 27, 300-302 (2002).

[12] A. Serov, B. Steinacher, and T. Lasser, "Full-field laser Doppler perfusion imaging and monitoring with an intelligent CMOS camera," Opt. Express 13, 3681-3689 (2005).

[13] A. Serov and T. Lasser, "High-speed laser Doppler perfusion imaging using an integrating CMOS image sensor," Opt. Express 13, 6416-6428, (2005.

[14] M. Draijer, E. Hondebrink, T. van Leeuwen, and W. Steenbergen, "Twente optical perfusion camera: system overview and performance for video rate laser Doppler perfusion imaging," Opt. Express 17, 3211-3225 (2009).

3зо [15] M. Leutenegger, E. Martin-Williams, P. Harbi, T. Thacher, W. Raffoul, M. André, A. Lopez, P. Lasser, and T. Lasser, "Real-time full field laser Doppler imaging," Biomed. Opt. Express 2, 1470-1477 (2011). 
[16] D. He, H. C. Nguyen, B. R. Hayes-Gill, Y. Zhu, J. A. Crowe, G. F. Clough, C. A. Gill, and S. P. Morgan, " $64 \times 64$ pixel smart sensor array for laser Doppler blood flow imaging," Opt. Lett. 37, 3060-3062 (2012).

[17] D. He, H. C. Nguyen, B. R. Hayes-Gill, Y. Zhu, J. A. Crowe, C. Gill, G. F. Clough, and S. P. Morgan, "Laser doppler blood flow imaging using a CMOS imaging sensor with on-chip signal processing," Sensors 13, 1263212647 (2013).

[18] P. Harbi and T. Thacher, "Body mapping of human cutaneous microcirculatory perfusion using a real-time laser Doppler imager," Diab. Vasc. Dis. Res. 10, 187-190 (2013).

[19] A. Ponticorvo and A. K. Dunn, "How to build a Laser Speckle Contrast Imaging (LSCI) system to monitor blood flow," J. Vis. Exp. 45, pii: 2004. doi: $10.3791 / 2004$.

[20] A. F. Fercher and J. D. Briers, "Flow visualization by means of singleexposure speckle photography," Opt. Comm. 37, 326-330 (1981).

[21] J. D. Briers and S. Webster, "Laser speckle contrast analysis (LASCA): a nonscanning, full-field technique for monitoring capillary blood flow," $J$. Biomed. Opt. 1, 174-179 (1996).

[22] J. D. Briers, "Laser Doppler, speckle and related techniques for blood perfusion mapping and imaging," Physiol. Meas. 22, R35-R66 (2001).

[23] K.R. Forrester, J. Tulip, C. Leonard, C. Stewart, and R.C. Bray, "A laser speckle imaging technique for measuring tissue perfusion," IEEE Trans. Biomed. Eng. 51, (2074-2084, (2004.

[24] J. D. Briers, "Laser speckle contrast imaging for measuring blood flow," Optica Applicata XXXVII, 139-152 (2007).

[25] M. Draijer, E. Hondebrink, T. van Leeuwen, and W. Steenbergen, "Review of laser speckle contrast techniques for visualizing tissue perfusion," Lasers Med. Sci. 24, 639-651 (2009). 
[26] D. A. Boas and A. K. Dunn, "Laser speckle contrast imaging in biomedical optics," J. Biomed. Opt. 15, article id. 011109 (2010).

[27] K. Basak, M. Manjunatha, and P. K. Dutta, "Review of laser speckle-based analysis in medical imaging," Med. Biol. Eng. Comput. 50, 547-558 (2012).

[28] J. Senarathna, A. Rege, N. Li, and N. V. Thakor, "Laser Speckle Contrast Imaging: theory, instrumentation and applications," IEEE Rev. Biomed. Eng. 6, 99-110 (2013).

[29] D. Briers, D. D. Duncan, E. Hirst, S. J. Kirkpatrick, M. Larsson, W. Steenbergen, T. Stromberg, and O. B. Thompson, "Laser speckle contrast imaging: theoretical and practical limitations," J. Biomed. Opt. 18, 66018 (2013).

[30] Perimed documentation (2009).

[31] O. B. Thompson and M. K. Andrews, "Tissue perfusion measurements: multiple-exposure laser speckle analysis generates laser Doppler-like spectra," J. Biomed. Opt., 15, 027015 (2010).

[32] P. Rousseau, G. Mahé, F. Haj-Yassin, S. Durand, A. Humeau, G. Leftheriotis, and P. Abraham, "Increasing the "region of interest" and "time of interest", both reduce the variability of blood flow measurements using laser speckle contrast imaging," Microvasc. Res. 82, 88-91 (2011).

[33] C. Puissant, P. Abraham, S. Durand, A. Humeau-Heurtier, S. Faure, G. Lefthériotis, P. Rousseau, and G. Mahé, "Reproducibility of non-invasive assessment of skin endothelial function using laser Doppler flowmetry and laser speckle contrast imaging," PLoS One 8, e61320, (2013).

[34] A. Humeau-Heurtier, P. Abraham, S. Durand, and G. Mahé, "Excellent inter- and intra-observer reproducibility of microvascular tests using laser speckle contrast imaging," Clin. Hemorheol. Microcirc., (2013) Nov 19. [Epub ahead of print]. 
[35] H. Mehrabian, R. Chopra, and A. L. Martel, "Calculation of intravascular signal in dynamic contrast enhanced-MRI using adaptive complex independent component analysis," IEEE Trans. Med. Imaging 32, 699-710 (2013).

[36] D. D. Duncan and S. J. Kirkpatrick, "Spatio-temporal algorithms for processing laser speckle imaging data," in Proc. Soc. Photo-opt. Ins. II 2008, $685802-1 / 6$.

[37] A. B. Parthasarathy, W. J. Tom, A. Gopal, X. Zhang, and A. K . Dunn, "Robust flow measurement with multi-exposure speckle imaging," Opt. Express 16, 1975-1989 (2008).

[38] P. Miao, M. Li, H. Fontenelle, A. Bezerianos, Y. Qiu, and S. Tong, "Imaging the cerebral blood flow with enhanced laser speckle contrast analysis (eLASCA) by monotonic point transformation," IEEE Trans. Biomed. Eng. 56, 1127-1133 (2009).

[39] D.D. Duncan, S.J. Kirkpatrick, "The copula: a tool for simulating speckle dynamics," JOSA A 25, 231-237 (2008).

[40] D.D. Duncan, S.J. Kirkpatrick, R.K. Wang, "Statistics of local speckle contrast" JOSA A 25, 9-15 (2008).

[41] F. Domoki, D. Zolei, O. Olah, V. Toth-Szuki, B. Hopp, F. Bari, and T. Smausz, "Evaluation of laser-speckle contrast image analysis techniques in the cortical microcirculation of piglets," Microvasc. Res. 83, 311-317 (2012).

[42] O. Yang and B. Choi, "Laser speckle imaging using a consumer-grade color camera," Opt. Lett. 37, 3957-3959 (2012).

[43] H. He, Y. Tang, F. Zhou, J. Wang, Q. Luo, and P. Li, "Lateral laser speckle contrast analysis combined with line beam scanning illumination to improve the sampling depth of blood flow imaging," Opt. Lett. 37, 37743776 (2012). 

wavelength laser speckle imaging to simultaneously access blood flow, blood volume, and oxygenation using a color CCD camera," Opt. Lett. 38, 36903692 (2013).

[45] T. Binzoni, A. Humeau-Heurtier, P. Abraham, and G. Mahe, "Blood perfusion values of laser speckle contrast imaging and laser Doppler flowmetry: is a direct comparison possible?," IEEE Trans. Biomed. Eng. 60, 1259-1265 (2013).

[46] L. M. Richards, S. M. Kazmi, J. L. Davis, K. E. Olin, and A. K. Dunn, "Low-cost laser speckle contrast imaging of blood flow using a webcam," Biomed. Opt. Express 4, 2269-2283 (2013).

[47] A. Humeau-Heurtier, P. Abraham, and G. Mahe, "Linguistic analysis of laser speckle contrast images recorded at rest and during biological zero: comparison with laser Doppler flowmetry data," IEEE Trans. Med. Imaging 32, 2311-2321 (2013).

${ }_{430}$ [48] S. M. Kazmi, S. Balial, and A. K. Dunn, "Optimization of camera exposure durations for multi-exposure speckle imaging of the microcirculation," Biomed. Opt. Express 5, 2157-2171 (2014).

[49] P. A. Oberg, "Laser-Doppler flowmetry," Crit. Rev. Biomed. Eng. 18, 125163 (1990).

[50] H. Cheng, Q. Luo, S. Zeng, S. Chen, J. Cen, and H. Gong, "Modified laser speckle imaging method with improved spatial resolution," J. Biomed. Opt. 8, 559-564 (2003).

[51] J. Yu, P. Miao, M. Li, Y. Qiu, Y. Zhu, and S. Tong, "High resolution mapping of cortical blood flow by mosaicing the laser speckle images," Conf. Proc. IEEE Eng. Med. Biol. Soc., 3743-3746 (2008). 
[52] S. Liu, P. Li, and Q. Luo, "Fast blood flow visualization of high-resolution laser speckle imaging data using graphics processing unit," Opt Express 16, 14321-14329 (2008).

[53] P. Miao, A. Rege, N. Li, N. V. Thakor, and S. Tong, "High resolution cerebral blood flow imaging by registered laser speckle contrast analysis," IEEE Trans. Biomed. Eng. 57, 1152-1157 (2010).

[54] M.A. Borkin, K.Z. Gajos, A. Peters, D. Mitsouras, S. Melchionna, F.J. Rybicki, C.L. Feldman, and H. Pfister, "Evaluation of artery visualizations for heart disease diagnosis," IEEE Trans. Vis Comput. Graph. 17(12), 2479-2488 (2011).

[55] T. Ropinski, S. Hermann, R. Reich, M. Schafers, and K. Hinrichs, "Multimodal vessel visualization of mouse aorta PET/CT scans," IEEE Trans. Vis Comput. Graph. 15(6), 1515-1522 (2009).

[56] H. Fujii, T. Asakura, K. Nohira, Y. Shintomi, and T. Ohura, "Blood flow observed by time-varying laser speckle," Opt. Lett. 10, 104-106 (1985).

[57] H. Fujii, K. Nohira, Y. Yamamoto, H. Ikawa, and T. Ohura, "Evaluation of blood flow by laser speckle image sensing. Part 1," Appl. Opt. 26, 53215325 (1987).

[58] M. Tekalp, Digital Video Processing, Prentice-Hall, New Jersey, 1995.

${ }_{460}$ [59] R. Arizaga, N. L. Cap, H. Rabal, and M. Trivi, "Display of local activity using dynamical speckle patterns," Opt. Eng. 41, 287-294 (2002).

[60] H. J. Rabal and R. A. Braga, Dynamic Laser Speckle and Applications, CRC Press, 2008.

[61] A. Zdunek, A. Adamiak, P. M. Pieczywek, and A. Kurenda, "The biospeckle method for the investigation of agricultural crops: A review," Opt. Lasers Eng. 52, 276-285 (2014). 
[62] L. Marti-Lopez, H. Cabrera, R. A. Martinez-Celorio, R. Gonzalez-Pena, "Temporal difference method for processing dynamic speckle patterns," Opt. Com. 283, 4972-4977 (2010).

[63] The recordings have been performed by N. Offenhauser and J. Dreier. Charité, Department of Experimental Neurology and Center for Stroke Research Berlin; Charitéplatz 1; 10117 Berlin; Germany.

[64] G. Mahe, F. Haj-Yassin, P. Rousseau, A. Humeau, S. Durand, G. Leftheriotis, and P. Abraham, "Distance between laser head and skin does not influence skin blood flow values recorded by laser speckle imaging," $\mathrm{Mi}$ crovasc. Res. 82, 439-442 (2011).

[65] J. C. Chang, K. C. Brennan, D. He, H. Huang, R. M. Miura, P. L. Wilson, and J. J. Wylie, "A mathematical model of the metabolic and perfusion effects on cortical spreading depression," PLoS One 8, e70469 (2013).

[66] V. E. Brunt and C. T. Minson, "KCa channels and epoxyeicosatrienoic acids: Major contributors to thermal hyperaemia in human skin," J. Physiol. 590, 3523-3534 (2012).

[67] N. Charkoudian, "Skin blood flow in adult human thermoregulation: How it works, when it does not, and why," in Mayo Clin. Proc. 78, 603-612 (2003)

[68] C. T. Minson, L. T. Berry, and M. J. Joyner, "Nitric oxide and neurally mediated regulation of skin blood flow during local heating," J. Appl. Physiol. 91, 1619-1626 (2001).

[69] J. Lu, E. Kazmierczak, J. H. Manton, and R. Sinclair, "Automatic segmentation of scaling in 2-D psoriasis skin images," IEEE Trans. Med. Imaging 32, 719-730 (2013). 


\section{Highlights}

- Laser speckle contrast imaging (LSCI) enables to monitor microvascular perfusion

- Large amount of data are often recorded leading to a long process of analysis

- Spatial averaging is therefore often performed in regions of interest (ROI)

- The spatial averaging reduces the spatial resolution of the images

- We propose a new visual representation of $\mathrm{LSCl}$ data using generalized differences 